\title{
Angiogenesis inhibitors for advanced hepatocellular carcinoma: in search for the right partner
}

\author{
Claudia Campani ${ }^{1}$, Lorenza Rimassa ${ }^{2,3}$, Nicola Personeni ${ }^{2,3}$, Fabio Marra ${ }^{1}$ \\ ${ }^{1}$ Department of Experimental and Clinical Medicine, Internal Medicine and Hepatology Unit, University of Florence, Florence, Italy; ${ }^{2}$ Medical \\ Oncology and Hematology Unit, Humanitas Cancer Center, Humanitas Clinical and Research Center-IRCCS, Rozzano, Milan, Italy; ${ }^{3}$ Department \\ of Biomedical Sciences, Humanitas University, Pieve Emanuele, Milan, Italy \\ Correspondence to: Fabio Marra, MD, PhD. Professor of Medicine, Department of Experimental and Clinical Medicine, University of Florence, Largo \\ Brambilla, 3, 50134 Florence, Italy. Email: fabio.marra@unifi.it. \\ Comment on: Abou-Alfa GK, Miksad RA, Tejani MA, et al. A Phase Ib, Open-Label Study of Dalantercept, an Activin Receptor-Like Kinase 1 Ligand \\ Trap, Plus Sorafenib in Advanced Hepatocellular Carcinoma. Oncologist 2019;24:161-e70.
}

Submitted May 11, 2020. Accepted for publication May 18, 2020.

doi: $10.21037 /$ atm-20-3788

View this article at: http://dx.doi.org/10.21037/atm-20-3788

Hepatocellular carcinoma (HCC) is the fifth most common cancer worldwide and one of the most frequent causes of cancer-related death. Systemic therapy is the standard treatment for advanced (BCLC-C) stage HCC and for patients with intermediate (BCLC-B) stage who are not suitable for hierarchically superior treatments (1). More than 10 years ago sorafenib, an angiogenesis inhibitor, was found to prolong survival in patients with advanced HCC (1). Sorafenib is a small molecule inhibitor which interferes with signaling of different receptors involved in angiogenesis, including vascular endothelial growth factor (VEGF)-R2, VEGF-R3 and platelet derived growth factor (PDGF)- $\beta$ R. For a long time, sorafenib has remained the only drug approved for systemic therapy of HCC, because of the failure, due to lack of efficacy or severe toxicity, of several phase III trials where alternative angiogenesis inhibitors have been tested (2). However, in the past three years the landscape of systemic therapy for HCC has been populated by additional drugs, which have been approved for first, second, and third-line treatment.

In the first-line setting, the phase III REFLECT trial showed that lenvatinib, a tyrosine kinase inhibitor (TKI) of VEGF-R1, VEGF-R2, VEGF-R3, fibroblast growth factor (FGF)-R1 to FGF-R4, PDGF- $\alpha$ R, KIT and RET, was non-inferior compared to sorafenib in terms of overall survival (OS) with an improvement in progression-free survival (PFS) (7.3 vs. 3.6 months), time to progression (TTP) (7.4 vs. 3.7 months), and objective response rate (ORR) (40.6\% vs. 12.4\%) (3). In the second-line setting, the phase III RESORCE trial enrolled patients who tolerated sorafenib but progressed on therapy (4) and demonstrated the efficacy and safety of regorafenib, a TKI of a wide spectrum of kinases including VEGFR, PDGFR, FGFR, TIE2, KIT, RET, and RAF. Treatment with regorafenib achieved a better OS (10.6 vs. 7.8 months), PFS, TTP, and ORR compared to placebo. Cabozantinib, an inhibitor of VEGF-R1, VEGF-R2, VEGF-R3, MET, and TAM family kinases, was tested in the phase III CELESTIAL trial, showing an advantage in OS (10.2 vs. 8.0 months), PFS, and ORR compared to placebo in patients previously treated with sorafenib, regardless of the tolerability. Of note, $30 \%$ of patients received cabozantinib or placebo as third-line treatment (5).

A peculiar aspect in systemic treatment of HCC is that almost none of the approved therapies is tailored based on the expression of cancer biomarkers. An attempt to personalize treatment was done with the phase III METIV-HCC trial that compared tivantinib to placebo in patients previously treated with sorafenib and with a high MET expression in tumor samples (6). However, this trial did not meet its primary end point of improvement in OS. Differently, positive results have been reported for ramucirumab, an anti-VEGFR2 monoclonal antibody, which improved OS (8.5 vs. 7.3 months) when used as second-line therapy in patients with baseline alphafetoprotein levels (AFP) $\geq 400 \mathrm{ng} / \mathrm{mL}$ (7).

Recently, immunotherapy has been applied to the treatment of HCC, and the FDA has approved nivolumab 
and pembrolizumab, two anti-PD-1 immune checkpoint inhibitors, as second-line treatments based on the positive results of two phase II trials $(8,9)$, the results of which led to two phase III trials. The CheckMate 459 trial compared nivolumab to sorafenib as first-line treatment, but no significant improvement in OS was observed (16.4 versus 14.7 months) (10). Moreover, in the KEYNOTE-240 trial, pembrolizumab as second-line treatment was not superior over placebo (13.9 vs. 10.6 months) (11). Nonetheless, these trials provided some positive signals that encourage further investigation of these novel therapeutic approaches in HCC.

Despite some good results in phase III trials, and a higher number of drugs available, the outcome of advanced HCC is still poor and side effects are numerous and relevant. Moreover, the mechanisms leading to HCC development are complex, and molecular classification reflects the heterogeneity of this tumor (12). For these reasons, a strategy that complements the action of angiogenesis inhibitors, targeting a different pathogenic mechanism within hepatocarcinogenesis is likely to produce synergistic effects and could result in better patient outcomes. An alternative strategy could be directed to more efficiently block the formation of new blood vessels. Because inhibition of the VEGF pathway is limited by intrinsic and acquired resistance, simultaneously targeting multiple steps involved in tumor angiogenesis could potentially overcome this resistance.

In a recent issue of The Oncologist, Abou-Alfa et al. reported the results of a phase Ib trial aiming to assess the safety and tolerability of dalantercept administered in combination with sorafenib, in patients with advanced HCC (13). Dalantercept (Figure 1) is a fully human recombinant fusion protein comprised of a human immunoglobulin G1 and the extracellular domain of the activin-like kinase (ALK)-1 receptor. ALK-1 belongs to the transforming growth factor (TGF)- $\beta$ receptor superfamily, and is mainly expressed on endothelial cells. Expression of ALK-1 has been reported on tumor blood vessels in lymphomas and in prostate, skin, thyroid, kidney, ovary, lung, pancreas and liver cancers (14). ALK-1 is a type I TGF- $\beta$ serine/threonine kinase receptor which binds with high affinity the ligands bone morphogenetic protein (BMP) 9 and 10, and forms a complex with a type II receptor such as BMP receptor II (14). This results in activation of Smad 1/5/8 signaling, formation of a complex with Smad4, and translocation to the nucleus, where cell type-specific molecules activate or repress the transcription of genes involved in various physiological processes. In endothelial cells, the DNA binding protein inhibitor ID-1 and transmembrane protein 100 (TMEM 100) are involved in the regulation of angiogenesis and lymphangiogenesis (15). Dalantercept functions as a ligand trap, preventing the binding of BMP9 and 10 to the ALK-1 receptor (Figure 1).

Angiogenesis is a tightly regulated process, involving different phases and orchestrated by a number of soluble factors. VEGF plays a pivotal role in the initial phase of the angiogenesis, stimulating proliferation and migration of endothelial cells and sprouting of endothelial tubes. Conversely, ALK-1 contributes to maturation and stabilization of early branched vessels, and its role in angiogenesis is demonstrated by several lines of evidence. Homozygous mutations of $A C V R L-1$, the gene encoding for ALK-1, cause embryonic death in mice because of the blockade of vascular development, and heterozygous mutations in humans cause hereditary hemorrhagic telangiectasia-2 (15). Generally, ALK-1 expression is suppressed in adults, but it can be induced in preexisting and new arterial vessels during wound healing and tumor angiogenesis (16).

As many patients have turned out to be resistant to drugs directed against VEGF or other pro-angiogenic factors, ALK-1 has been identified as an additional pathway to be possibly targeted for cancer treatment. The NCT02024087 trial reported in The Oncologist was justified by the results of preclinical and early clinical evidence demonstrating a possible antitumor effect of ALK-1 inhibition. PF03446962, a monoclonal antibody directed against ALK-1 $(16,17)$ interferes with BMP9/10 signaling and also blocks recruitment of endoglin, a coreceptor, to the receptor complex (18). PF-03446962 led to significant tumor growth inhibition in xenograft models of breast cancer or melanoma (18). Support to the possible therapeutic role of ALK-1 inhibition in HCC has been provided by a phase I study investigating the effects of PF-03446962 as a single agent in patients previously treated with anti-angiogenic therapy. In half of the 24 patients enrolled, stable disease was observed, lasting 12 weeks or longer in seven patients. Thrombocytopenia (33\%), fatigue (29\%), and chills (17\%) were among the most common treatment-related adverse events. Of note, patients with disease control tended to have higher serum BMP9 levels and lower levels of TGF- $\beta$ and VEGF-R3 than patients with progressive disease. Despite a modest activity of PF-03446962 monotherapy, these data set the stage for treatment with diverse ALK-1 inhibitors.

With respect to dalantercept, an earlier phase I 


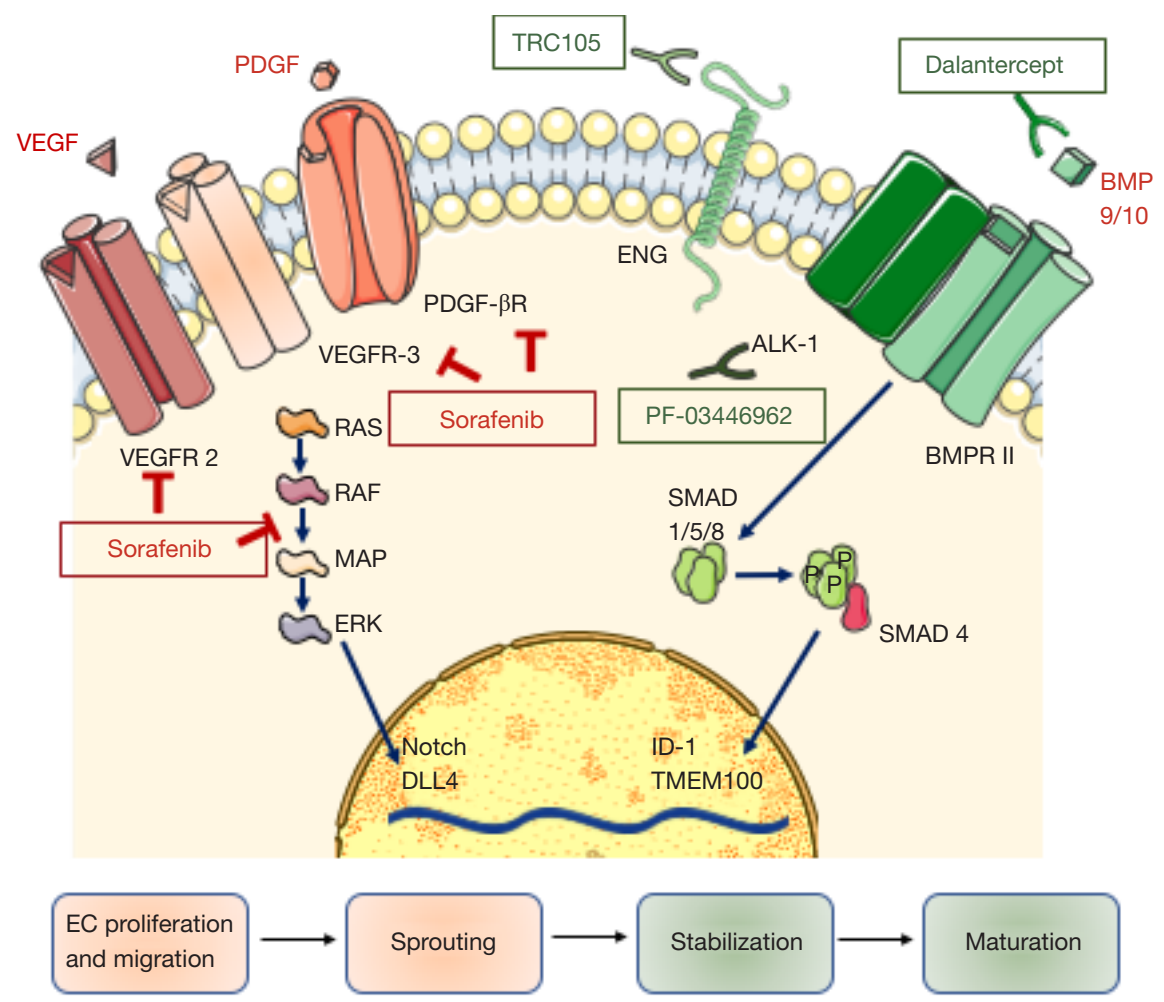

Figure 1 Therapeutic approaches targeting tumor angiogenesis. VEGF and PDGF stimulate proliferation and migration of endothelial cells, and sprouting of tubular structures through their cognate receptors (activation phase). These receptors activate multiple signaling pathways, including Ras-ERK and downstream Notch and DLL. BMP 9/10 promote the stabilization of vessels, with reconstitution of a basement membrane and the recruitment of pericytes, developing functional vascular beds (maturation phase). BMP 9/10 bind BMPR II (a type II TGF- $\beta$ receptor) which phosphorylates ALK-1 and promotes the formation of a receptor complex together with endoglin, a coreceptor. This complex leads to phosphorylation and activation of SMADs $1 / 5 / 8$, which bind to SMAD 4 and translocate to the nucleus leading to expression of various genes such as ID-1 and TMEM100. The figure mainly illustrates receptors and pathways affected by drugs discussed herein. Sorafenib interferes with signaling of different receptors and with the Ras/ERK pathway. Dalantercept is a 'ligand trap' preventing binding of BMP9/10 to the ALK-1 receptor. PF-03446962 blocks ALK-1 activation. TRC105 interferes with endoglin. ALK1: Activin like kinase-1; BMPR II: type II TGF $\beta$ receptor; BMP: bone morphogenetic protein; ID-1: DNA-binding protein inhibitor, TMEMT100: transmembrane protein 100; ENG: endoglin; EC endothelial cell; VEGF-R: vascular endothelial growth factor receptor; PDGF: platelet derived growth factor

trial enrolled 37 patients with advanced solid cancers, including one with HCC. Patients received dalantercept at escalating doses $(0.1-4.8 \mathrm{mg} / \mathrm{kg})$ every 3 weeks until disease progression, and an expansion cohort was treated with 0.8 or $1.6 \mathrm{mg} / \mathrm{kg}$ every 3 weeks until disease progression. One patient had a partial response, and 13 patients had stable disease, 8 of whom for more than 12 weeks. Interestingly, the response was associated with reduced tumor blood flow and appearance of telangiectasia $(19,20)$. The promising results of this trial led to the development of phase II trials of dalantercept as monotherapy, with a recommended dose of $1.2 \mathrm{mg} / \mathrm{kg}$, in ovarian, head and neck, and endometrial cancers (20-22). Dur to unsatisfactory results, alternative strategies combining the effects of ALK-1 inhibition with those of 'conventional' angiogenesis inhibitors have been tested. Of note, since no clinically relevant hypertension, proteinuria, gastrointestinal perforations, or hemorrhage were observed in a prior phase I study (19), from a safety standpoint dalantercept appears to be an optimal partner for schedules that involve concurrent VEGF pathway inhibitors. The rationale behind such associations is to block vascular proliferation with a VEGF receptor inhibitor, 
blocking at the same time vessel maturation and stabilization promoted by the activation of the ALK-1 receptor. This strategy was recently tested in pretreated patients with renal cell carcinoma, where dalantercept was administered in association with axitinib, an anti-VEGF TKI, in a phase II study (23). Dalantercept was administered at 0.6, 0.9, or $1.2 \mathrm{mg} / \mathrm{kg}$ subcutaneously every 3 weeks plus axitinib $5 \mathrm{mg}$. The ORR was $25 \%$ and the overall median PFS was 8.3 months. Seven patients obtained a partial response, 17 had stable disease, and 14 patients achieved disease control for more than six months.

Based on these and preclinical findings (24) the effects of the combination of dalantercept and sorafenib were explored in patients with HCC in the phase Ib trial published in The Oncologist (13). Twenty-one patients with HCC naive to systemic therapy were enrolled. Whilst sorafenib dose was fixed at $400 \mathrm{mg}$ qd in all patients, 5 patients received subcutaneous dalantercept at $0.6 \mathrm{mg} / \mathrm{kg}$ and the remaining 16 patients received dalantercept at $0.4 \mathrm{mg} / \mathrm{kg}$. This dose reduction was necessary due to severe peripheral edema and one dose-limiting toxicity, namely grade 4 hyponatremia. After dose reduction, no adverse events of grade $>3$ were reported. The study showed that the dalantercept/sorafenib combination was well tolerated, although dalantercept had to be administered at a lower dose (23). As a matter of fact, the question arises as to whether a biologically effective dalantercept concentration could be achieved in the study on HCC patients (13). In addition, sorafenib was administered at half of the recommended dose when used as a single agent, and this is in contrast with other phase I studies in HCC where the full dose was used in combination with other agents. Importantly, no safety concerns emerged in a recently completed study testing sorafenib plus the humanized antiendoglin monoclonal antibody TRC105. Also, preliminary results suggested potential efficacy deriving from this sorafenib/TRC105 combination (25).

The Authors reported that the study did not meet its secondary endpoint of proving a convincing antitumor activity (13). Overall survival was markedly variable, ranging from 1.9 to 23.3 months, and it was compared with the median OS of 10.7 months (95\% CI: 9.4-13.3) reported for sorafenib in the SHARP trial (26). However, no information on the median OS, nor TTP were provided. From the published paper we learn that no complete or partial responses were achieved, while $56 \%$ of patients had stable disease, although it is unclear for how long this lasted. A point that needs to be underscored is the lack of information about the possible presence of underlying cirrhosis or liver disease in the patients enrolled in the trial. Furthermore, information on PFS, BCLC stage, AFP levels, and etiologies is lacking. As a final assessment, the Authors concluded that the level of antitumor activity did not meet the planned endpoint.

Although the data reported by Abou-Alfa et al. (13) were considered negative, and the program for testing dalantercept in HCC has been interrupted, these results allow to make several interesting considerations on the future of combination therapies in HCC. At this time, the combination of antiangiogenetic agents with immune checkpoint inhibitors appears to be the most promising strategy. The rationale for these associations is related to the evidence that cancer cells have a greater expression of PD-L1 when HIF-1 expression is increased due to hypoxia. Moreover, hypoxia is responsible for increased VEGF expression, that plays an inhibitory effect on the immune system, blocking the maturation of dendritic cells and favoring the priming of immunosuppressive inflammatory cell subsets (2). Accordingly, VEGF is felt to drive the reprogramming of cancer-associated fibroblasts, tumorassociated macrophages, and tumor-associated endothelial cells (27). These events will eventually determine the lineage commitment within the hepatic microenvironment.

Currently, several phase I-II-III trials have been designed to study this association. The combination of atezolizumab and bevacizumab as a first line treatment has been studied in untreated patients with locally advanced or metastatic HCC and well-preserved liver function. Improved OS and PFS compared to sorafenib and a twice as high ORR (32\%) were reported (28). These results make this study the first positive phase III immunotherapy trial, and the first positive phase III combination trial in HCC, and shows for the first time the superiority of a new first-line treatment compared to sorafenib in HCC.

Two further phase III trials evaluating the association of immune checkpoint inhibitors and antiangiogenic drugs are ongoing. The LEAP-002 trial (29) evaluates the efficacy and safety of lenvatinib in combination with pembrolizumab as first-line therapy in patients with advanced HCC and well-preserved liver function. This study started after the promising results of a phase $\mathrm{Ib}$ trial where the combination of lenvatinib and pembrolizumab obtained a good safety profile and an ORR of $48 \%$. COSMIC-312 is a phase III trial which compares the combination of cabozantinib and atezolizumab $v s$. sorafenib $v s$. cabozantinib as firstline treatment in patients with advanced HCC (30). An additional rationale of this study is linked to the evidence 


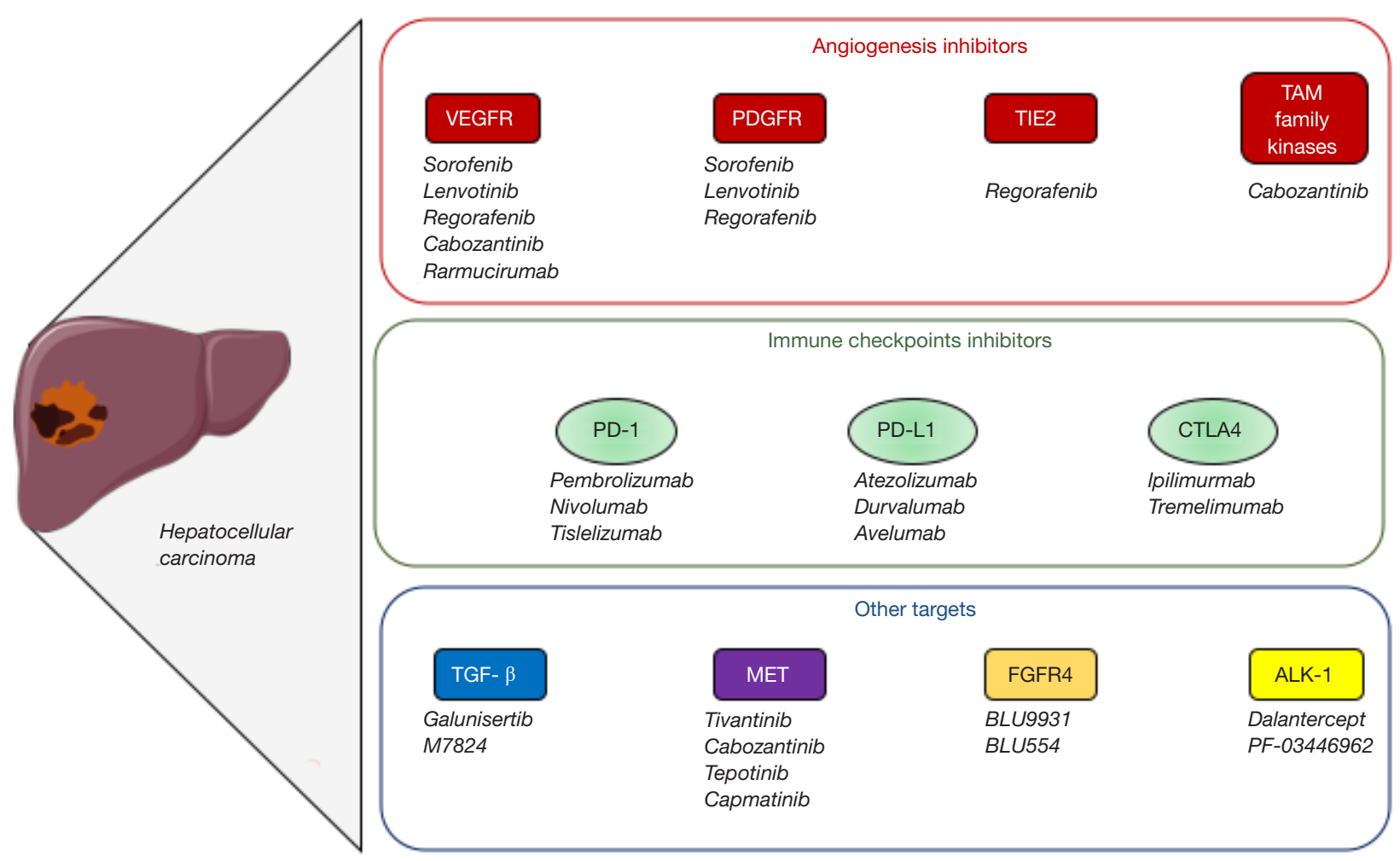

Figure 2 Major targets of systemic therapy in HCC. Angiogenesis inhibitors (top panel) have been approved as monotherapy for systemic treatment of HCC (for further details please refer to text). Immune checkpoint inhibitors (middle panel) are currently being investigated in association with approved angiogenesis inhibitors, or with other drugs (e.g., bevacizumab) interfering with angiogenesis. The lower panel illustrates additional targets under investigation as monotherapy or in combination with other molecules. HCC, hepatocellular carcinoma.

that cabozantinib, differently from other TKIs, has a direct action on the immune system, promoting an immune permissive environment. Indeed, cabozantinib increases the expression of major histocompatibility complex class 1 antigen, promoting greater sensitivity of tumor cells to $\mathrm{T}$ cell-mediated killing (2). Interestingly, these new lines of evidence could possibly put again into play the ALK1 system, because in a mouse model of breast cancer, TRC105 was more effective in immunocompetent mice, in a $\mathrm{T}$ cell dependent manner, as depletion of CD4 or CD8 cells abrogated the effects of ENG inhibition (15). These data show a possible crosstalk between the ALK-1/endoglin pathway and the antitumoral immune machinery.

While the combination of anti-angiogenics and immune checkpoint inhibitors is driving most of the attention, additional targets for HCC treatment (Figure 2) are being explored (2). These are tested as single agents but have the potential to be combined with other more established drugs in the field, such as modulators of angiogenesis or of the immune system. The time is promising for making a decisive leap in the systemic treatment of HCC. However, it should be kept in mind that information on the molecular characteristics of the tumor have not yet been incorporated into clinical practice. It is necessary that all upcoming studies require a liver biopsy performed immediately before entering the trial, and possibly a higher number of patients routinely treated in the clinic should undergo biopsy to make more solid correlations between the molecular characteristics of the tumor, the presence of targets, and the ultimate patient outcome.

\section{Acknowledgments}

Work on liver cancer of Dr.Marra's group is supported by grants from the Associazione Italiana per la Ricerca sul Cancro (AIRC), Cassa di Risparmio di Pistoia e Pescia, and from the University of Florence. Figures were made in part with SMART (Servier Medical AT, by Servier ${ }^{\circledR}$ ), 
which is licensed under a Creative Commons Attribution 3.0 Unreported License: https://smart.servier.com/ Funding: None.

\section{Footnotes}

Provenance and Peer Review: This article was commissioned by the editorial office, Annals of Translational Medicine. The article did not undergo external peer review.

Conflicts of Interest: The authors have completed the ICMJE uniform disclosure form (available at http:// dx.doi.org/10.21037/atm-20-3788). LR reports grants and personal fees from Lilly, personal fees from Bayer, personal fees from Sirtex Medical, personal fees and nonfinancial support from ArQule, grants and personal fees from Exelixis, grants, personal fees and nonfinancial support from Ipsen, personal fees from Celgene, grants and personal fees from Eisai, grants and personal fees from AstraZeneca, personal fees from AbbVie, personal fees from Gilead, personal fees from Roche, personal fees from Hengrui Therapeutics, grants and personal fees from MSD, personal fees from Baxter, personal fees from Amgen, personal fees from Italfarmaco, personal fees from Sanofi, grants and personal fees from Incyte, personal fees from Basilea, grants from Agios, grants from ARMO Biosciences, grants from Beigene, outside the submitted work; NP reports personal fees from ArQule, personal fees from Gilead, personal fees from AbbVie, outside the submitted work; FM reports personal fees and non-financial support from Abbvie, personal fees from Allergan, personal fees and nonfinancial support from Bayer, personal fees from Gilead, personal fees from Ipsen, personal fees from Intercept, personal fees from Menarini, personal fees from Novo Nordisk, non-financial support from Alfa-Wasserman, outside the submitted work. The other author has no conflicts of interest to declare.

Ethical Statement: The authors are accountable for all aspects of the work in ensuring that questions related to the accuracy or integrity of any part of the work are appropriately investigated and resolved.

Open Access Statement: This is an Open Access article distributed in accordance with the Creative Commons Attribution-NonCommercial-NoDerivs 4.0 International License (CC BY-NC-ND 4.0), which permits the noncommercial replication and distribution of the article with the strict proviso that no changes or edits are made and the original work is properly cited (including links to both the formal publication through the relevant DOI and the license). See: https://creativecommons.org/licenses/by-nc-nd/4.0/.

\section{References}

1. EASL Clinical Practice Guidelines: Management of hepatocellular carcinoma. J Hepatol 2018;69:182-236.

2. Faivre S, Rimassa L, Finn RS. Molecular therapies for HCC: Looking outside the box. J Hepatol 2020;72:342-52.

3. Kudo M, Finn RS, Qin S, et al. Lenvatinib versus sorafenib in first-line treatment of patients with unresectable hepatocellular carcinoma: a randomised phase 3 noninferiority trial. Lancet 2018;391:1163-73.

4. Bruix J, Qin S, Merle P, et al. Regorafenib for patients with hepatocellular carcinoma who progressed on sorafenib treatment (RESORCE): a randomised, double-blind, placebo-controlled, phase 3 trial. Lancet 2017;389:56-66.

5. Abou-Alfa GK, Meyer T, Cheng AL, et al. Cabozantinib in Patients with Advanced and Progressing Hepatocellular Carcinoma. N Engl J Med 2018;379:54-63.

6. Rimassa L, Assenat E, Peck-Radosavljevic M, et al. Tivantinib for second-line treatment of MET-high, advanced hepatocellular carcinoma (METIV-HCC): a final analysis of a phase 3, randomised, placebo-controlled study. Lancet Oncol 2018;19:682-93.

7. Zhu AX, Kang YK, Yen CJ, et al. Ramucirumab after sorafenib in patients with advanced hepatocellular carcinoma and increased $\alpha$-fetoprotein concentrations (REACH-2): a randomised, double-blind, placebocontrolled, phase 3 trial. Lancet Oncol 2019;20:282-96.

8. Zhu AX, Finn RS, Edeline J, et al. Pembrolizumab in patients with advanced hepatocellular carcinoma previously treated with sorafenib (KEYNOTE-224): a non-randomised, open-label phase 2 trial. Lancet Oncol 2018;19:940-52.

9. El-Khoueiry AB, Sangro B, Yau T, et al. Nivolumab in patients with advanced hepatocellular carcinoma (CheckMate 040): an open-label, non-comparative, phase 1/2 dose escalation and expansion trial. Lancet 2017;389:2492-502.

10. Yau T, Park JW, Finn R, et al. CheckMate 459: A randomized, multi-center phase III study of nivolumab (NIVO) vs sorafenib (SOR) as first-line (1L) treatment in patients (pts) with advanced hepatocellular carcinoma (aHCC). Ann Oncol 2019;30:V874-5.

11. Finn RS, Ryoo BY, Merle P, et al. Pembrolizumab As Second-Line Therapy in Patients With Advanced 
Hepatocellular Carcinoma in KEYNOTE-240: A Randomized, Double-Blind, Phase III Trial. J Clin Oncol 2020;38:193-202.

12. Dhanasekaran R, Nault JC, Roberts LR, et al. Genomic Medicine and Implications for Hepatocellular Carcinoma Prevention and Therapy. Gastroenterology 2019;156:492-509.

13. Abou-Alfa GK, Miksad RA, Tejani MA, et al. A Phase Ib, Open-Label Study of Dalantercept, an Activin ReceptorLike Kinase 1 Ligand Trap, plus Sorafenib in Advanced Hepatocellular Carcinoma. Oncologist 2019;24:161-e70.

14. Cunha SI, Pietras K. ALK1 as an emerging target for antiangiogenic therapy of cancer. Blood 2011;117:6999-7006.

15. Bhatt RS, Atkins MB. Molecular pathways: can activin-like kinase pathway inhibition enhance the limited efficacy of VEGF inhibitors? Clin Cancer Res 2014;20:2838-45.

16. Seki T, Yun J, Oh SP. Arterial endothelium-specific activin receptor-like kinase 1 expression suggests its role in arterialization and vascular remodeling. Circ Res 2003;93:682-9.

17. Simonelli M, Zucali P, Santoro A, et al. Phase I study of PF-03446962, a fully human monoclonal antibody against activin receptor-like kinase-1, in patients with hepatocellular carcinoma. Ann Oncol 2016;27:1782-7.

18. Hu-Lowe DD, Chen E, Zhang L, et al. Targeting activin receptor-like kinase 1 inhibits angiogenesis and tumorigenesis through a mechanism of action complementary to anti-VEGF therapies. Cancer Res 2011;71:1362-73.

19. Bendell JC, Gordon MS, Hurwitz HI, et al. Safety, pharmacokinetics, pharmacodynamics, and antitumor activity of dalantercept, an activin receptor-like kinase-1 ligand trap, in patients with advanced cancer. Clin Cancer Res 2014;20:480-9.

20. Makker V, Filiaci VL, Chen LM, et al. Phase II evaluation of dalantercept, a soluble recombinant activin receptor-like kinase 1 (ALK1) receptor fusion protein, for the treatment of recurrent or persistent endometrial cancer: an NRG Oncology/Gynecologic Oncology Group Study 0229N. Gynecol Oncol 2015;138:24-9.

21. Jimeno A, Posner MR, Wirth LJ, et al. A phase 2 study

Cite this article as: Campani C, Rimassa L, Personeni N, Marra F. Angiogenesis inhibitors for advanced hepatocellular carcinoma: in search for the right partner. Ann Transl Med 2020;8(22):1532. doi: 10.21037/atm-20-3788 of dalantercept, an activin receptor-like kinase-1 ligand trap, in patients with recurrent or metastatic squamous cell carcinoma of the head and neck. Cancer 2016;122:3641-9.

22. Burger RA, Deng W, Makker V, et al. Phase II evaluation of dalantercept in the treatment of persistent or recurrent epithelial ovarian cancer: An NRG Oncology/Gynecologic Oncology Group study. Gynecol Oncol 2018;150:466-70.

23. Voss MH, Bhatt RS, Plimack ER, et al. The DART Study: Results from the Dose-Escalation and Expansion Cohorts Evaluating the Combination of Dalantercept plus Axitinib in Advanced Renal Cell Carcinoma. Clin Cancer Res 2017;23:3557-65

24. Wang X, Solban N, Khanna P, et al. Inhibition of ALK1 signaling with dalantercept combined with VEGFR TKI leads to tumor stasis in renal cell carcinoma. Oncotarget 2016;7:41857-69.

25. Duffy AG, Ma C, Ulahannan SV, et al. Phase I and Preliminary Phase II Study of TRC105 in Combination with Sorafenib in Hepatocellular Carcinoma. Clin Cancer Res 2017;23:4633-41.

26. Llovet JM, Ricci S, Mazzaferro V, et al. Sorafenib in advanced hepatocellular carcinoma. N Engl J Med 2008;359:378-90.

27. Ma L, Hernandez MO, Zhao Y, et al. Tumor Cell Biodiversity Drives Microenvironmental Reprogramming in Liver Cancer. Cancer Cell 2019;36:418-30.e6.

28. Chen AL, Qin S, Ikeda M, et al. IMbrave 150: Efficacy and safety results from a ph III study evaluating atezolizumab (ate) - Bevacizumab (ben) vs sorafenib (Sor) as first treatment (tx) for patients (its) with unresectable hepatocellular carcinoma (HCC). Ann Oncol 2019;30:186-7.

29. Llovet JM, Kudo M, Cheng AL, et al. Lenvatinib (len) plus pembrolizumab (pembro) for the first-line treatment of patients (pts) with advanced hepatocellular carcinoma (HCC): Phase 3 LEAP-002 study. J Clin Oncol 2019;37:4152.

30. Kelley R, Cheng AL, Braiteh F, et al. Phase 3 (COSMIC-312) study of cabozantinib (C) in combination with atezolizumab (A) versus sorafenib (S) in patients (pts) with advanced hepatocellular carcinoma (aHCC) who have not received previous systemic anticancer therapy. Ann Oncol 2019;30:4157. 